\title{
Effect of chitosan combined with hyaluronate on promoting the recovery of postoperative facial nerve regeneration and function in rabbits
}

\author{
HUAWEI LIU ${ }^{1}$, HAITAO HUANG ${ }^{2}$, WENTING BI ${ }^{3}$, XINYING TAN ${ }^{1}$, RUNXIN LI ${ }^{1}$, WEISHENG WEN ${ }^{1}$, \\ WENLING SONG ${ }^{4}$, YANHUA ZHANG ${ }^{4}$, FENG ZHANG ${ }^{4}$ and $\mathrm{MIN} \mathrm{HU}^{1}$ \\ ${ }^{1}$ Department of Stomatology, Chinese People's Liberation Army General Hospital, Beijing 100853; \\ ${ }^{2}$ Department of Stomatology, The 1st Affiliated Hospital of Dalian Medical University, Dalian, Liaoning 116011; \\ ${ }^{3}$ Department of Stomatology, Beijing First Hospital of Integrated Chinese and Western Medicine, Beijing 100026; \\ ${ }^{4}$ Department of Quality Control, Beijing Yierkang Bioengineering Development Center, Beijing 102600, P.R. China
}

Received March 26, 2017; Accepted March 29, 2018

DOI: $10.3892 / \mathrm{etm} .2018 .6238$

\begin{abstract}
To determine better solutions for postoperative nerve functional recovery, the effects of chitosan and hyaluronate on perineural scar formation and neural function recovery were investigated in 40 rabbits. Rabbits were randomized into 4 groups: A (chitosan), B (chitosan + hyaluronate), C (hyaluronate) and $\mathrm{D}$ (control). The rabbits underwent the same parotidectomy surgery, but different materials were used to cover the operated nerves. By evaluating specific indicators, including vibrissae motion tests, neural electrophysiological examinations and extraneural examinations, it was revealed that the amplitude of vibrissae motion of all groups had increased 6 weeks after surgery. The recovery of Group B was superior compared with all other groups at 4 and 12 weeks post-surgery; however no significant differences were detected. Group B exhibited a great number of nerve fibers, thicker myelin sheath and greater nerve conduction velocity. In summary, the use of a chitosan conduit combined with sodium hyaluronate gel may prevent perineural scar formation in facial nerves and promote nerve functional recovery.
\end{abstract}

\section{Introduction}

Compression, blunt or transection injuries caused by continuous compression of the peripheral nerve axons damage the normal structure of nerve axons (1). During axonal recovery and regeneration, fibroblasts secrete large amounts of collagen, resulting in the formation of perineural scars (2). Scars tissue may form an intraneural barrier, which hinders

Correspondence to: Dr Min Hu, Department of Stomatology, Chinese People's Liberation Army General Hospital, 28 Fuxing Road, Beijing 100853, P.R. China

E-mail: humin48@vip.163.com

Key words: chitosan, facial nerve, neural scar, hyaluronate the regeneration of nerve axons and may induce tissue adhesion, inhibiting the neural blood supply and resulting in neural ischemia (3). The aim of the present study was to determine a method to improve the prevention of perineural scarring and scarring of surrounding tissues due to facial nerve injuries following parotidectomy surgeries. This novel method may improve postoperative nerve functional recovery.

Chitosan is a type of natural polymeric biological polysaccharide with higher biodegradability and biocompatibility compared with a number of other scar prevention materials (including, human amniotic membrane and Seprafilm) $(4,5)$; chitosan can be automatically degraded into glucosamine in vivo and absorbed by humans (6). Additionally, its mechanical properties (including, tensile strength and compressive strength) make chitosan an ideal extracellular matrix material (7). Hyaluronate is a vital component of the extracellular matrix, which is widely expressed in animals (8). Hyaluronate has previously been used in many in vivo studies due to its availability, non-toxic biodegradability and because it did not trigger immunological rejection $(2,9)$. To the best of our knowledge, there is currently no experimental study that has investigated the perineural scar prevention of facial nerves. The aim of the present study was to investigate the effect of chitosan combined with hyaluronate on the formation of perineural scars and nerve functional recovery in a model of facial nerve injury.

\section{Materials and methods}

Animals and surgical methods

Experimental animals. A total of 40 adult (12 months old) New Zealand white female rabbits (weight, 2.5-3.0 kg) were included in the current study. The rabbits were supplied by the Laboratory Animal Centre of Chinese PLA General Hospital (Beijing, China) and maintained under controlled conditions: 1 atmosphere, $20-25^{\circ} \mathrm{C}, 12 \mathrm{~h}$ light/dark cycle and humidity of 40-60\%. The rabbits were given access to food and water ad libitum. Rabbits were randomized into four groups (n=10/group): A (chitosan), B (chitosan + hyaluronate), 
C (hyaluronate) and D (control). Anesthesia procedures, painless surgeries and euthanasia procedures were approved by the ethics committee of the Chinese People's Liberation Army General Hospital (Beijing, China). Rabbits were treated according to the requirements of the local animal ethics commission.

Preparation of chitosan conduit. The chitosan conduits (Beijing Yierkang Bioengineering Development Center, Beijing, China) were disinfected with ${ }^{60} \mathrm{Co}$ (inner diameter, $1.8 \mathrm{~mm}$; tube wall thickness, $0.05 \mathrm{~mm}$ ) and immersed into normal saline for $10 \mathrm{~min}$ at room temperature prior to surgery. The chitosan conduits were cut into sections (length, 1 or $0.5 \mathrm{~cm}$; circumference, $0.5 \mathrm{~cm}$ ) and split longitudinally. Chitosan conduits ( $1 \mathrm{~cm}$ length) were used to cover upper and lower buccal branches of the facial nerve. Chitosan conduits (length, $0.5 \mathrm{~cm}$ ) were also used to cover cervical and zygomatic branches of the facial nerve.

Surgical methods. Rabbits were anesthetized via intramuscular injections $(50 \mathrm{mg} / \mathrm{kg}$ ketamine $+5 \mathrm{mg} / \mathrm{kg}$ xylazine hydrochloride) and immobilized in a lateral position with the right side of their faces shaved. The buccal branch of the facial nerve was exposed by a $4-\mathrm{cm}$-long incision between the tragus and the lower margin of the mandible. The facial nerve trunk, upper and lower buccal branches and cervical and zygomatic branches were fully exposed and separated from the basement membrane. Complete resection of the superficial lobe and major resection of the deep lobe of the parotid gland were conducted and the residual tissue was sutured using 4-0 sutures.

In Group A, the facial nerves were covered by chitosan conduits (Fig. 1). In Group B, the nerves were covered by chitosan conduits and then evenly coated with hyaluronate on the surface $(0.2 \mathrm{ml} ; 15 \mathrm{mg} / \mathrm{ml}$; Shanghai Medical Instruments Co., Ltd., Shanghai, China). In Group C, the surfaces of the nerves were evenly coated with $0.2 \mathrm{ml}$ hyaluronate. In Group D, the nerves were exposed and did not receive treatments.

Following the establishment of the model, the tissue was sutured and a rubber drainage strip was indwelled. The wound was disinfected for 3 days using iodophor (once/day) and penicillin was injected intramuscularly for 3 days $(0.06 \mathrm{~g} / \mathrm{kg}$, twice/day). The drainage strips were indwelled for 2-3 days until no exudation was observed. Rabbits were euthanized intravenously with pentobarbital sodium at the dose of $100 \mathrm{mg} / \mathrm{kg}$ 12 weeks after surgery.

\section{Postoperative examination}

Vibrissae motion evaluation. At $24 \mathrm{~h}$ and 2, 4, 6, 8, 10 and 12 weeks after surgery, the vibrissae motion on the surgical side was compared with that of the contralateral side. Motion was scored using the following criteria: 0 , no motion with vibrissae hanging down; 1 , minor movement; 2 , inadequate movement; 3 , adequate movement, but weaker compared the contralateral side; 4 , normal movement, identical to the contralateral side $(10,11)$.

Scar adhesion analysis. At 4 and 12 weeks after surgery, the incision was open again under anesthesia. According to the classification standard by Petersen, the force of separating the skin
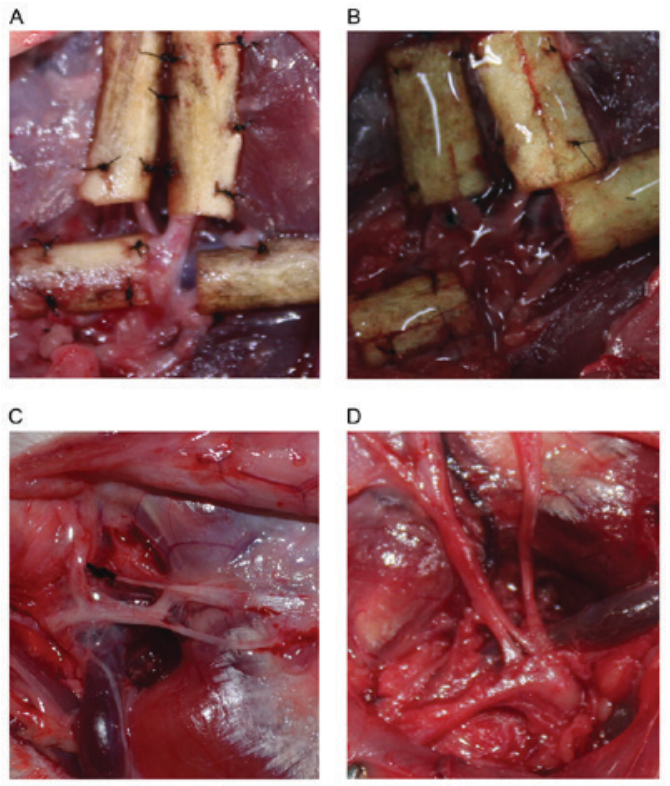

Figure 1. Surgical exposure of facial nerves. The facial nerves of a rabbit treated with (A) chitosan conduit, (B) chitosan conduit and hyaluronate, and (C) hyaluronate. (D) The facial nerves of a rabbit in the control group.

from muscles and fascia from nerves was graded as follows: i) Separation was not required or only blunt dissection was required; ii) hard blunt separation was required; and iii) sharp separation was required (10).

Electrophysiological evaluation. Under general anesthesia at 4 and 12 weeks after surgery, the former incision site was opened and the facial nerve trunk and branches were exposed. Two stimulating electrodes were placed on the trunk, while the recording electrode was inserted into the orbicularis oris. The amplitude and latency of the orbicularis oris, and the distance between two electrodes was measured. The nerve conduction velocity (NCV) of the branches was calculated using the following formula: $\mathrm{v}=(\mathrm{d} 1-\mathrm{d} 2) /(\mathrm{t} 1-\mathrm{t} 2)$, where $\mathrm{d} 1-\mathrm{d} 2$ was the distance between the two electrodes and $\mathrm{t} 1$ - $\mathrm{t} 2$ was the spike latency of the orbicularis oris excited by two stimulating electrodes.

Histological staining. Following the electrophysiological examinations, the chitosan conduits or nerve samples were removed from 5 rabbits for each time point (postoperative weeks 4 and 12), the conduits were then divided into $\sim 4$ sections and fixed in paraffin or epoxy.

i) Extraneural scar examination. One of the middle sections was fixed in $10 \%$ formaldehyde for $24 \mathrm{~h}$ at $4^{\circ} \mathrm{C}$, embedded in paraffin, sliced into 5- $\mu \mathrm{m}$-thick sections and subjected to Masson staining for $24 \mathrm{~h}$ at $4^{\circ} \mathrm{C}$ to assess the status of extraneural scar hyperplasia and the amount of type I and III collagen fibers. The sections were observed using a light microscopy with a magnification of $\mathrm{x} 200$. Four visual fields were observed to measure the average collagen thickness of extraneural scars.

The other middle section was fixed directly at $4^{\circ} \mathrm{C}$ in $2.5 \%$ glutaric acid for $24 \mathrm{~h}$ and $1 \%$ osmic acid for $2 \mathrm{~h}$, sliced into semi-thin $(2-\mu \mathrm{m}$-thick) sections, dehydrated in an ascending series of acetone and embedded in epoxy resin at $4^{\circ} \mathrm{C}$ for 
$10 \mathrm{~min}$. Harris hematoxylin staining was conducted at $4^{\circ} \mathrm{C}$ for $10 \mathrm{~min}$ and tissues were observed under a transmission electron microscopy (TEM) at a magnification of x3,000. The average thickness of collagen in the epineurium and conduit were measured in six optical fields per section.

ii) Axon morphometric analysis. The epoxy-embedded sections were sliced into semi-thin (2- $\mu$ m-thick) sections and the number of myelinated fibers was measured under an inverted microscope in six random optical fields per section. The sections were also sliced into ultrathin (50-nm-thick) sections. The thickness of the myelin sheath, and the number and diameter of myelinated fibers were observed in six optical fields per section.

Conduit analysis. At 12 weeks after surgery, implanted chitosan conduits were removed, fixed in $2.5 \%$ glutaric acid for $24 \mathrm{~h}$ at $4^{\circ} \mathrm{C}$, allowed to dry out and adhered to a metal plate with conductive tapes. Next, the samples were introduced into the chamber of the sputter coater and coated with a very thin film of gold. The surface and cross section of the conduits were investigated prior to and 12 weeks after implantation under a scanning electron microscope (SEM) at a magnification of x500.

Statistical analysis. Statistical analysis was performed using SPSS 19.0 (IBM Corp., Armonk, NY, USA). Data are presented as the mean \pm standard deviation. Analysis of variance was used to compare differences among groups followed by the Student-Newman-Keuls test. $\mathrm{P}<0.05$ was considered to indicate a statistically significant difference.

\section{Results}

Vibrissae motion is similar among the four groups. No significant differences in vibrissae motion were identified between $24 \mathrm{~h}$ and 2 weeks post-surgery in any group (Fig. 2). At week 4, scores were higher in Groups B and C compared with Groups A and D, but no statistical differences were identified. From week 6, vibrissae motion was markedly improved in all groups. Group D had the lowest scores, however no significant differences were identified.

Scarring is greatest in control rabbits 12 weeks after surgery. At week 4, the nerves had only mildly adhered to the surrounding tissue in all groups and were easy to separate (Fig. 3). No significant differences were identified between the groups at week 4, although Groups B and $\mathrm{C}$ had the lowest scores according to the Petersen classification system. At week 12 after surgery, Group D adhered to the surrounding tissue significantly more compared with the other groups $(\mathrm{P}<0.05)$. No significant differences were identified among the experimental groups.

Epineuria are thicker in control rabbits 12 weeks after surgery. At week 4, the epineurium was thicker in Group D compared with the treatment groups. However, no significant differences were identified. At week 12, the thickness of the epineurium in Group D was significantly greater compared with the treatment groups $(\mathrm{P}<0.01$; Figs. 4 and 5$)$.

Axon morphology analysis. H\&E staining and TEM analyses were performed to observe the morphology of the nerve fibers.

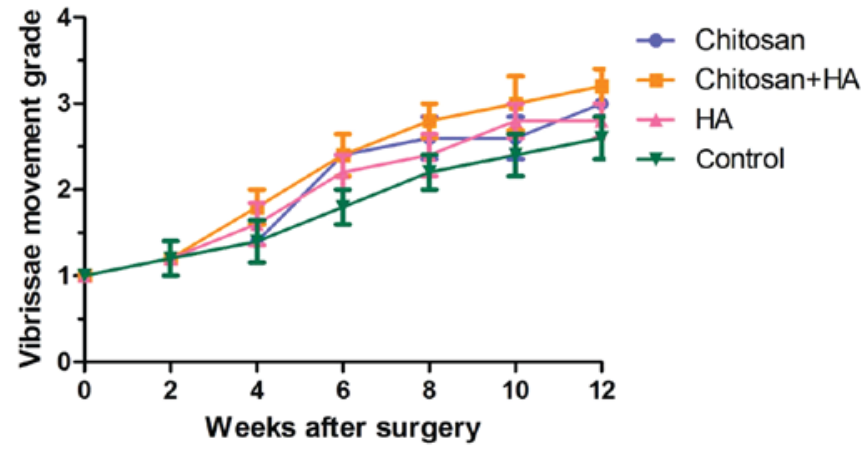

Figure 2. Vibrissae motion is similar among the four groups. Vibrissae motion on the surgical side was compared with that of the contralateral side $24 \mathrm{~h}$ and 2, 4, 6, 8, 10 and 12 weeks after surgery. HA, hyaluronate.

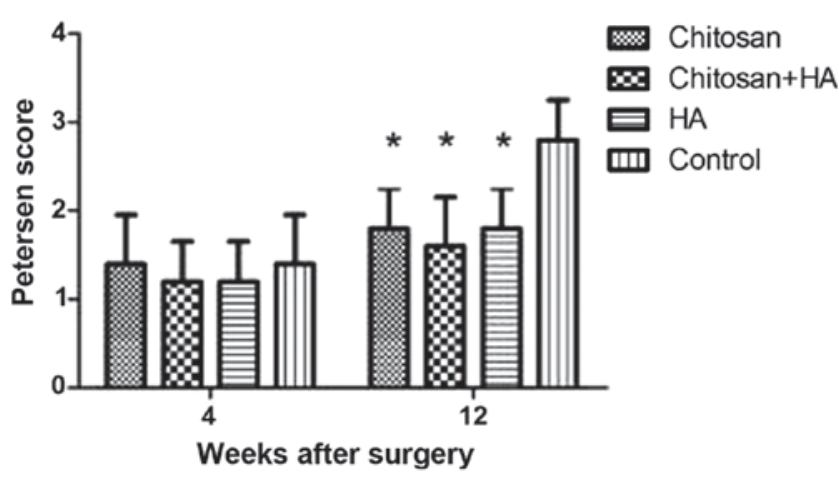

Figure 3. Scarring was greatest in control rabbits 12 weeks after surgery. Scar adhesion was assessed at week 4 and 12 according to the classification standard by Petersen. ${ }^{*} \mathrm{P}<0.05$ vs. the control group. HA, hyaluronate.
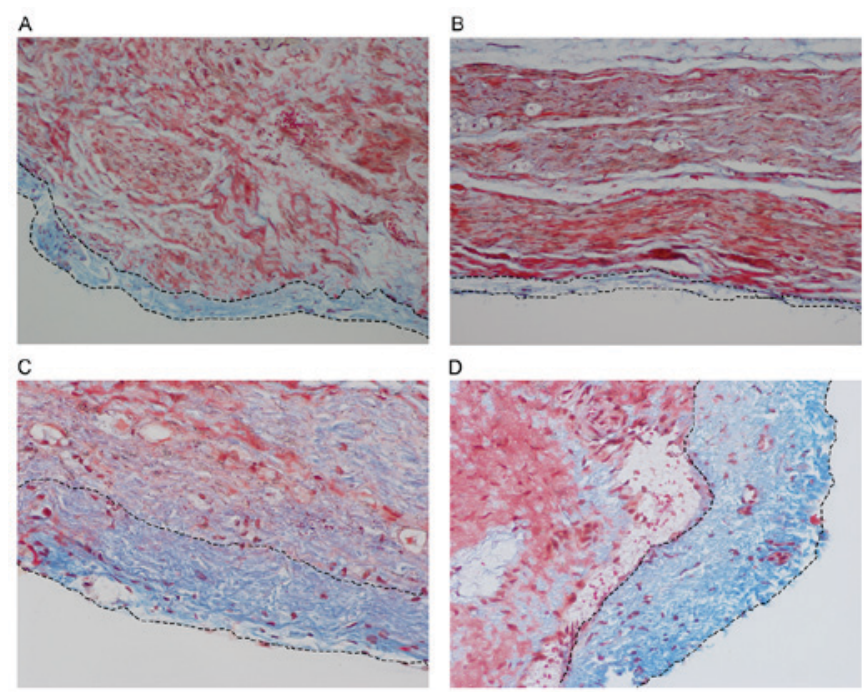

Figure 4. Epineuria are thinner with chitosan conduit or chitosan conduit and hyaluronate combination treatment. (A) Chitosan group, (B) chitosan + hyaluronate group, (C) hyaluronate group and (D) control group. Masson staining was performed 12 weeks after surgery. The blue stain between the dotted lines represents the epineurium, which consists primarily of collagen. Magnification, $\mathrm{x} 200$.

The number and diameter of nerve fibers and the myelin sheath thickness were quantified using the images from the TEM analysis. 


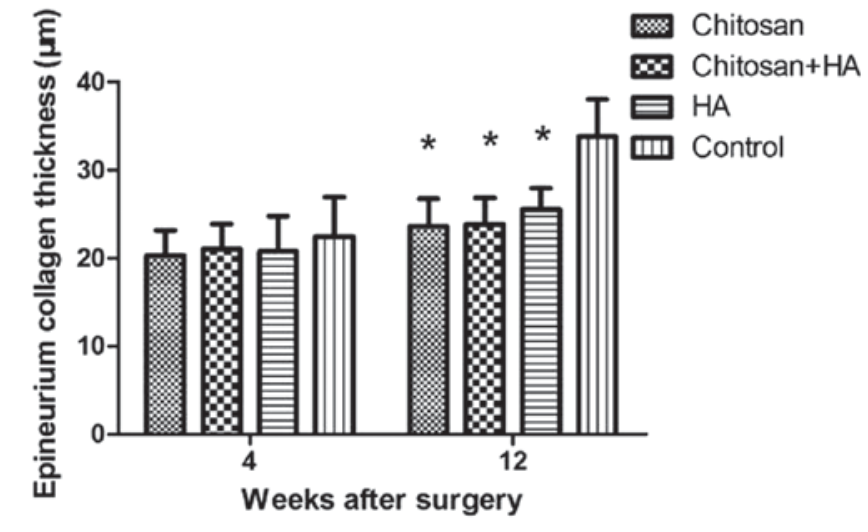

Figure 5. Epineuria are thicker in control rabbits 12 weeks after surgery. Masson staining was performed. The thicknesses of the epineuria were quantified at 4 and 12 weeks after surgery. ${ }^{*} \mathrm{P}<0.01$ vs. the control group. HA, hyaluronate.

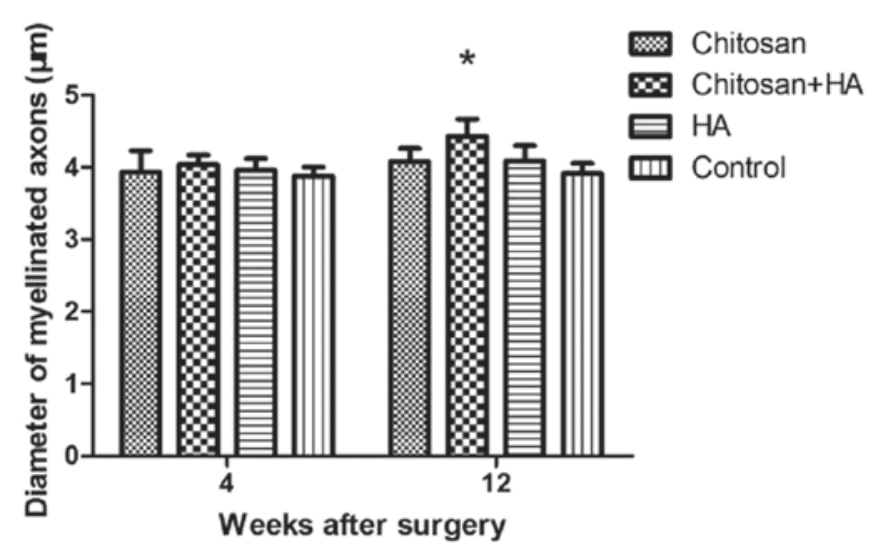

Figure 6. Myelinated fiber diameters were greatest with chitosan conduit and HA treatment at 12 weeks after surgery. Myelinated fiber diameters were determined by microscopy with a transmission electron microscope and quantified 4 and 12 weeks after surgery. ${ }^{*} \mathrm{P}<0.05$ vs. the control group. HA, hyaluronate.

Myelinated nerve fiber diameter is larger with chitosan and hyaluronate treatment. At 4 weeks, no significant differences in the mean diameter of myelinated nerve fibers were observed between the groups. However, at 12 weeks post-surgery, the mean diameter of myelinated nerve fibers in Group B was significantly larger compared with Group D $(\mathrm{P}<0.05$; Fig. 6).

Myelinated nerve fiber numbers are greater with chitosan and hyaluronate treatment. At 4 weeks following surgery, no significant differences in the number of myelinated nerve fibers were identified between the groups. However, the number of myelinated nerve fibers in Group B was significantly greater compared with Group D at week $12(\mathrm{P}<0.05$; Fig. 7).

Myelin sheath thickness increases with chitosan and hyaluronate treatment. At 4 weeks following surgery, no significant differences in myelin sheath thickness were identified between the groups. However, the myelin sheath thickness in Group B was significantly increased compared with Group D at week 12 ( $\mathrm{P}<0.05$; Fig. 8).

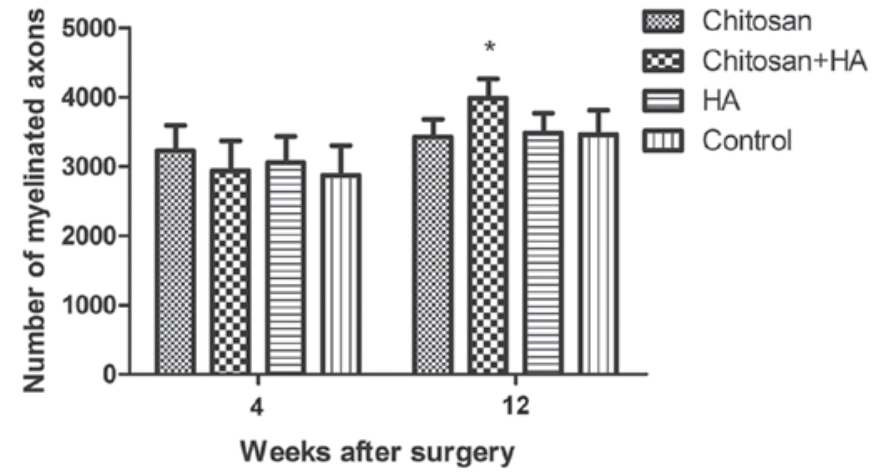

Figure 7. A greater number of myelinated nerve fibers were observed with chitosan conduit and HA treatment 12 weeks after surgery. The myelinated fibers were assessed by microscopy with a transmission electron microscope, and quantified 4 and 12 weeks after surgery. ${ }^{*} \mathrm{P}<0.05$ vs. the control group. HA, hyaluronate.

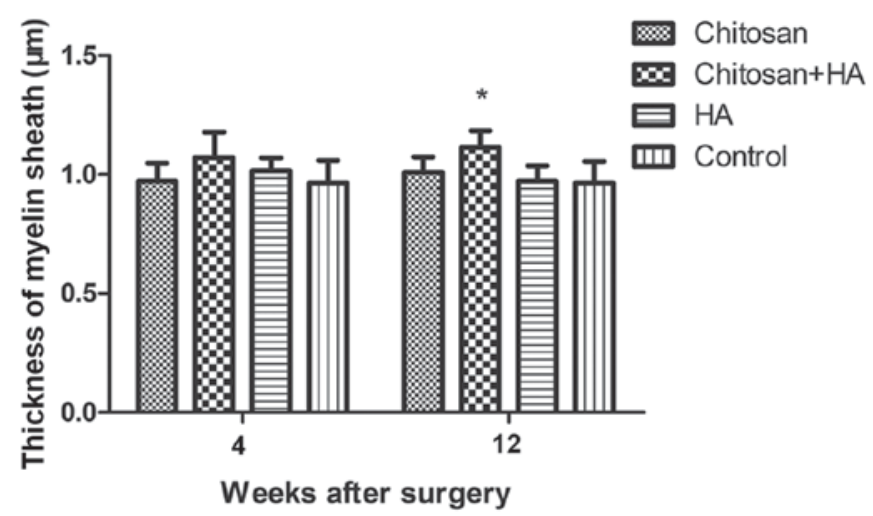

Figure 8. Myelin sheath thicknesses were observed with chitosan conduit and HA treatment 12 weeks after surgery. The thicknesses of the Schwann cells were determined by microscopy with a transmission electron microscope and quantified at 4 and 12 weeks after surgery. ${ }^{*} \mathrm{P}<0.05$ vs. the control group. HA, hyaluronate.

Conduit analysis. Prior to and at 12 week after surgery, the surface and cross section of the chitosan conduits were observed using SEM. At week 12, chitosan conduits had partially been degraded and absorbed; also, diameters of the chitosan conduits pores had increased and the conduits had been partially absorbed into the nerves (Fig. 9). At week 12 after surgery, chitosan conduits were revealed to be compatible with the rabbits as they were partially absorbed and no regional inflammatory rejection occurred.

Neural electrophysiological measurement. NCV was measured by electrophysiological assessment; at week 4 post-surgery, no significant differences in NCV were identified between the groups. At week 12, NCV in Group B was significantly faster compared with Group D ( $\mathrm{P}<0.05$; Fig. 10).

\section{Discussion}

Severe injury to peripheral nerves causes extraneural scarring, which increases the adhesion of nerves to surrounding tissues (12). The adhesion of peripheral nerves to surrounding tissues can lead to compressive edema within nerve bundles, 

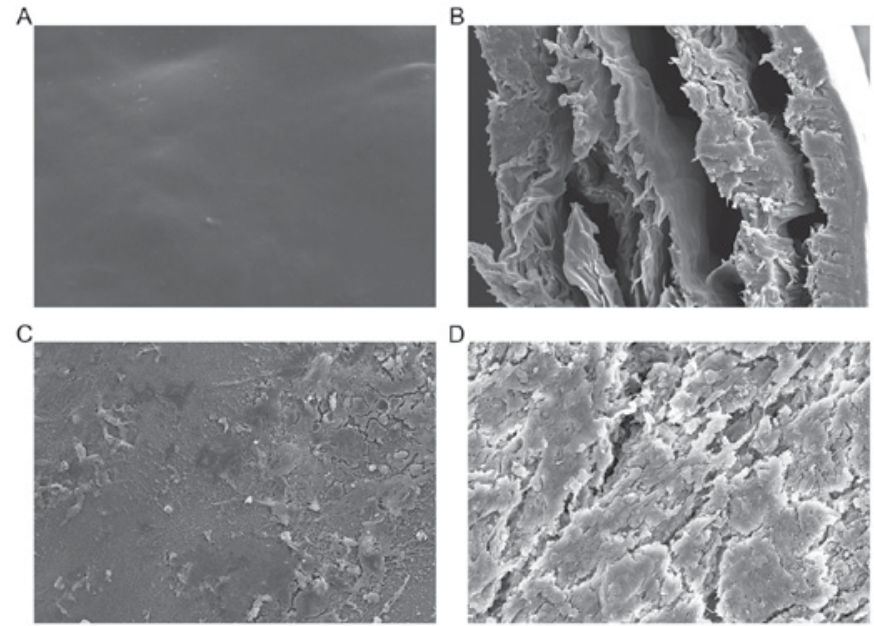

Figure 9. Implanted chitosan conduits observed under a scanning electron microscope. The (A) surface and (B) cross section of a chitosan conduit prior to implantation. The (C) surface and (D) cross-section of a chitosan conduit 12 weeks after implantation. Magnification, x 500 .

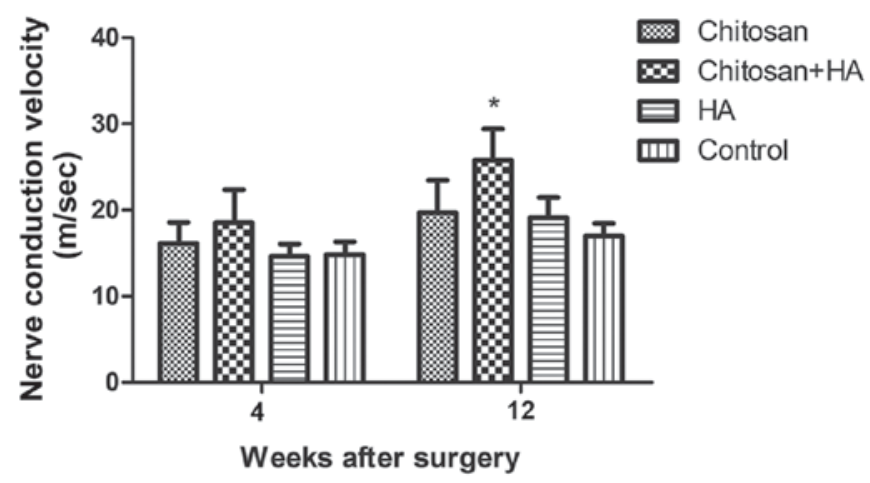

Figure 10. Neural conduction velocity was fastest with chitosan conduit and HA treatment 12 weeks after surgery. Nerve conduction velocity was determined by electrophysiological evaluation and quantified 4 and 12 weeks after surgery. ${ }^{*} \mathrm{P}<0.05$ vs. the control group. HA, hyaluronate.

fibrotic changes and tethering; fibrotic changes can interfere with blood flow to the nerve and tethering can be harmful when stretching the extremities (13). Therefore, extraneural scarring has an impact on the functional recovery of peripheral nerves.

Several studies have demonstrated that using a variety of physical barriers or pharmaceutical interventions can promote peripheral nerve regeneration and functional recovery $(2,5,9,14-16)$. Peripheral nerve scar formation is associated with damage to the blood-nerve barrier structure and function and fibroblast activation, as well as extracellular matrix and cytokine imbalance conversion regulation disorders $(17,18)$.

Thus, reducing the number of activated fibroblasts, downregulating $\alpha$-smooth muscle actin, reducing transforming growth factor (TGF)- $\beta 1$ and TGF- $\beta 2$, increasing TGF- $\beta 3$ and increasing the activity of collagenase have been used to inhibit the generation of peripheral nerve scars (19). A number of reagents have been demonstrated to reduce peripheral nerve scar production and promote the recovery of neurological function, including upregulating TGF- $\beta$ inhibitors mannose-6-phosphate, tissue plasminogen activator, chondroitinase, interleukin-10, adrenocorticotropic hormone, hyaluronate, human amniotic fluid, placenta suspension and heparin sulfate glycosaminoglycan $(9,20-24)$. However, to the best of our knowledge, no studies have investigated perineural scar prevention of the facial nerve.

Facial nerve injury following parotid gland surgery or injury often requires surgical exposure of the facial nerve (25). In the present study, damage to the facial nerve and surrounding tissue with the potential to cause facial nerve peripheral scarring and affect the restoration of facial nerve function was inflicted. Such damage often leads to different degrees of facial paralysis, affecting the patient's physical and mental health (25). In revision surgery for recurrent parotid gland tumors, adhesion of the facial nerve to the surrounding tissue increases the difficulty of the surgery and makes protecting the facial nerve more challenging (26). It has been reported that the incidence of permanent facial paralysis in the first parotid gland surgery was $0-3.9 \%$, however this could rise to $11.3-29.0 \%$ in revision surgery (27-29).

Chitosan is a natural polysaccharide macromolecule, which can inhibit the growth of fibroblasts and prevent tissue adhesion $(2,30)$. The compatibility and hydrophilicity of chitosan are improved following carboxymethylation, making it suitable for implantation in vivo (2). High-degree deacetylation of chitosan can inhibit the contraction of collagen-producing fibroblasts, which may serve an important role in alleviating scar formation (31). As a natural substance in vivo, hyaluronate is biodegradable and serves as a neurolytic agent to reduce scarring $(2,32)$.

In the present study, chitosan conduits combined with hyaluronate was selected to investigate improvements in neural regeneration during nerve repair. The potential preventive effects of chitosan, hyaluronate or a combination on extraneural scarring and adhesion were evaluated using a rat facial nerve injury model. A total of 4 weeks after surgery, no scarring was identified in the nerves or surrounding tissues of the three experimental groups, with infrequent adherence of muscle and nerve tissues. Nerve membrane collagen thickness was lower in the treatment groups compared with the control, however this difference was not statistically significant. Furthermore, the Petersen scores of Groups B and C were lower compared with Group D. Scar adhesion formation was markedly improved at week 12 compared with week 4 in all groups. The Petersen score of Group D was significantly higher and the collagen in the external scar tissue was thicker compared with the other three groups.

It was hypothesized that the scar tissue began to form and wrap around the nerves of Group C and D at week 4. Continuative compression may induce degeneration of axons and myelin sheaths, which may affect the recovery of nerve function (33). The initial formation of fibrosis takes 2-3 weeks, but the maturation process may last for 9 months $(34,35)$. At 4 weeks post-surgery, fibrosis had not been fully completed, so the specimen was able to be isolated easily. At week 12, however, the fibrosis process appeared to be complete as the nerves were significantly more difficult to dissect from the surrounding tissues in Group D compared with the experimental groups. It was hypothesized that chitosan conduits effectively prevented neurosurgical scar hyperplasia as it inhibited the secretion of collagen from activated fibroblasts. Park et al (2) demonstrated 
that a hyaluronic acid-carboxymethylcellulose solution could reduce the adhesion of nerves to surrounding tissues and prevent the formation of scars. The current study also revealed that hyaluronate could inhibit scar formation and that a chitosan conduit in conjunction with hyaluronate may be more efficient. Ishikawa et al (36) demonstrated that chitosan conduits could promote nerve regeneration following sciatic nerve defects.

In the current study, 12 weeks after surgery the number of myelinated nerve fibers, the thickness of the myelin sheath and NCV of Groups B were greater compared with Groups C and $\mathrm{D}$. The myelinated nerve fiber diameter, number of myelinated nerve fibers, myelin sheath thickness and NCV of Group B were significantly higher compared with Group D. It was determined that neural scarring constricted the nerves continuously in Groups A, C and D, which may have degenerated the axons and myelin sheaths. Neural scarring may hinder the recovery of neurological function. These findings further demonstrate that chitosan conduits combined with hyaluronate may improve facial nerve regeneration and function.

In summary, chitosan conduits and/or hyaluronate promote the recovery of postoperative facial nerve function in rabbits. The main limitation of the present study was the small sample size used, resulting in a number of non-significant differences. Future studies involving additional nerves and larger samples are warranted.

As biocompatible absorbable materials, chitosan conduits and hyaluronate have several advantages, including a wide variety of sources and the possibility of mass production. In addition, the combination of a chitosan conduit and hyaluronate was more effective in preventing perineural scar production and promoting facial nerve regeneration and functional recovery following parotidectomy surgeries.

\section{Acknowledgements}

Not applicable.

\section{Funding}

The current study was supported by grants from the National Natural Science Foundation of China (grant no. 81371116) and Science and Technology Innovation Fund of the Chinese People's Liberation Army General Hospital (grant no. 16KMM16).

\section{Availability of data and materials}

All data generated or analyzed during this study are included in this published article.

\section{Authors' contributions}

HL and WB analyzed the data. HL, HH and XT performed the animal experiments. WB, RL, WS, YZ and FZ performed the tissue staining. WW and $\mathrm{MH}$ designed the experiments.

\section{Ethics approval and consent to participate}

Animal anesthesia and euthanasia procedures were approved by the Ethics Committee of the Chinese People's Liberation Army General Hospital (Beijing, China).

\section{Consent for publication}

Not applicable.

\section{Competing interests}

The authors declare that they have no competing interests.

\section{References}

1. Lane JM, Bora FW Jr and Pleasure D: Neuroma scar formation in rats following peripheral nerve transection. J Bone Joint Surg Am 60: 197-203, 1978.

2. Park JS, Lee JH, Han CS, Chung DW and Kim GY: Effect of hyaluronic acid-carboxymethyl cellulose solution on perineural scar formation after sciatic nerve repair in rats. Clin Orthop Surg 3: 315-324, 2011.

3. Dy CJ, Aunins B and Brogan DM: Barriers to Epineural scarring: Role in treatment of traumatic nerve injury and chronic compressive neuropathy. J Hand Surg Am 43: 360-367, 2018.

4. Ozgenel GY and Filiz G: Combined application of human amniotic membrane wrapping and hyaluronic acid injection in epineurectomised rat sciatic nerve. J Reconstr Surg 20: 153-157, 2004.

5. Magill CK, Tuffaha SH, Yee A, Luciano JP, Hunter DA, Mackinnon SE and Borschel GH: The short- and long-term effects of Seprafilm on peripheral nerves: A histological and functional study. J Reconstr Microsurg 25: 345-354, 2009.

6. Bak M, Gutkowska ON, Wagner E and Gosk J: The role of chitin and chitosan in peripheral nerve reconstruction. Polim Med 47: 43-47, 2017.

7. Fregnan F, Ciglieri E, Tos P, Crosio A, Ciardelli G, Ruini F, Tonda-Turo C, Geuna S and Raimondo S: Chitosan crosslinked flat scaffolds for peripheral nerve regeneration. Biomed Mater 11: 045010, 2016.

8. Marcuzzo AV, Tofanelli M, Boscolo Nata F, Gatto A and Tirelli G: Hyaluronate effect on bacterial biofilm in ENT district infections: A review. APMIS 125: 763-172, 2017.

9. Smit X, van Neck JW, Afoke A and Hovius SE: Reduction of neural adhesions by biodegradable autocrosslinked hyaluronic acid gel after injury of peripheral nerves: An experimental study. J Neurosurg 101: 648-652, 2004.

10. Petersen J, Russell L, Andrus K, MacKinnon M, Silver J and Kliot M: Reduction of extraneural scarring by ADCON-T/N after surgical intervention. Neurosurgery 38: 976-984, 1996.

11. Most SP: Facial nerve recovery in bcl 2 overexpression mice after crush injury. Arch Facial Plast Surg 6: 82-87, 2004.

12. Shintani K, Uemura T, Takamatsu K, Yokoi T, Onode E, Okada $\mathrm{M}$ and Nakamura $\mathrm{H}$ : Protective effect of biodegradable nerve conduit against peripheral nerve adhesion after neurolysis. J Neurosurg 20: 1-10, 2017.

13. Gaudet AD, Popovich PG and Ramer MS: Wallerian degeneration: Gaining perspective on inflammatory events after peripheral nerve injury. J Neuroinflammation 8: 110, 2011.

14. Abe Y, Doi K and Kawai S: An experimental model of peripheral nerve adhesion in rabbits. Br J Plast Surg 58: 533-540, 2005.

15. Xu J, Varitimidis SE, Fisher KJ, Tomaino MM and Sotereanos DG: The effect of wrapping scarred nerves with autogenous vein graft to treat recurrent chronic nerve compression. J Hand Surg Am 25: 93-103, 2000.

16. Albayrak BS, Ismailoglu O, Ilbay K, Yaka U, Tanriover G, Gorgulu A and Demir N: Doxorubicin for prevention of epineurial fibrosis in a rat sciatic nerve model: Outcome based on gross postsurgical, histopathological, and ultrastructural findings. J Neurosurg Spine 12: 327-333, 2010.

17. Ahmed Z, Dent RG, Leadbeater WE, Smith C, Berry M and Logan A: Matrix metalloproteases: Degradation of the inhibitory environment of the transected optic nerve and the scar by regenerating axons. Mol Cell Neurosci 28: 64-78, 2005.

18. Kim JS, Choi IG, Lee BC, Park JB, Kim JH, Jeong JH, Jeong JH and Seo CH: Neuregulin induces CTGF expression in hypertrophic scarring fibroblasts. Mol Cell Biochem 365: 181-189, 2012.

19. Zou T, Ling C, Xiao Y, Tao X, Ma D, Chen ZL, Strickland S and Song H: Exogenous tissue plasminogen activator enhances peripheral nerve regeneration and functional recovery after injury in mice. J Neuropathol Exp Neurol 65: 78-86, 2006. 
20. Ngeow WC, Atkins S, Morgan CR, Metcalfe AD Boissonade FM, Loescher AR and Robinson PP: The effect of Mannose-6-phosphate on recovery after sciatic nerve repair. Brain Res 1394: 40-48, 2011

21. Neubauer D, Graham JB and Muir D: Chondroitinase treatment increases the effective length of acellular nerve grafts. Exp Neurol 207: 163-170, 2007.

22. Atik B, Erkutlu I, Tercan M, Buyukhatipoglu H, Bekerecioglu M and Pence S: The effects of exogenous melatonin on peripheral nerve regeneration and collagen formation in rats. J Surg Res 166 330-336, 2011.

23. Atkins S, Loescher AR, Boissonade FM, Smith KG, Occleston N, O'Kane S, Ferguson MW and Robinson PP: Interleukin-10 reduces scarring and enhances regeneration at a site of sciatic nerve repair. J Peripher Nerv Syst 12: 269-276, 2007.

24. Dam-Hieu P, Lacroix C, Said G, Devanz P, Liu S and Tadie M: Reduction of postoperative perineural adhesions by Hyaloglide gel: An experimental study in the rat sciatic nerve. Neurosurgery 56 (2 Suppl): S425-S433, 2005.

25. Glas AS, Vermey A, Hollema H, Robinson PH, Roodenburg JL, Nap RE and Plukker JT: Surgical treatment of recurrent pleomorphic adenoma of the parotid gland: A clinical analysis of 52 patients. Head Neck 23: 311-316, 2001.

26. Romano A, Cama A, Corvino R, Graziano P, Friscia M Iaconetta $\mathrm{G}$ and Califano L: Complications after parotid gland surgery Our experience. Ann Ital Chir 88: 295-301, 2017.

27. Wittekindt C, Streubel K, Arnold G, Stennert E and GuntinasLichius O: Recurrent pleomorphic adenoma of the parotid gland: Analysis of 108 consecutive patients. Head Neck 29: 822-828, 2007.

28. Liu H, Wen W, Huang H, Liang Y, Tan X, Liu S, Liu C and Hu M: Recurrent pleomorphic adenoma of the parotid gland: Intraoperative facial nerve monitoring during parotidectomy. Otolaryngol Head Neck Surg 151: 87-91, 2014.

29. Dell'Aversana Orabona G, Bonavolonta P, Iaconetta G, Forte R and Califano L: Surgical management of benign tumors of the parotid gland: Extracapsular dissection versus superficial parotidectomy-our experience in 232 cases. J Oral Maxillofac Surg 71: 410-413, 2013.
30. Wu XB, Peng CH, Huang F, Kuang J, Yu SL, Dong YD and Han BS: Preparation and characterization of chitosan porous microcarriers for hepatocyte culture. Hepatobiliary Pancreat Dis Int 10: 509-515, 2011

31. Howling GI, Dettmar PW, Goddard PA, Hampson FC, Dornish M and Wood EJ: The effect of chitin and chitosan on fibroblast-populated collagen lattice contraction. Biotechnol Appl Biochem 36: 247-253, 2002.

32. Ikeda K, Yamauchi D, Osamura N, Hagiwara N and Tomita K: Hyaluronic acid prevents peripheral nerve adhesion. Br J Plast Surg 56: 342-347, 2003.

33. Li BH, Yang $\mathrm{K}$ and Wang X: Biodegradable magnesium wire promotes regeneration of compressed sciatic nerves. Neural Regen Res 11: 2012-2017, 2016.

34. Soller EC, Tzeranis DS, Miu K, So PT and Yannas IV: Common features of optimal collagen scaffolds that disrupt wound contraction and enhance regeneration both in peripheral nerves and in skin. Biomaterials 33: 4783-4791, 2012.

35. Demir R, Yayla M, Akpinar E, Cakir M, Calikoglu C, Ozel L, Ozdemir G and Mercantepe T: Protective effects of alpha-lipoic acid on experimental sciatic nerve crush injury in rats: Assessed with functional, molecular and electromicroscopic analyses. Int J Neurosci 124: 935-943, 2014

36. Ishikawa N, Suzuki Y, Ohta M, Cho H, Suzuki S, Dezawa M and Ide C: Peripheral nerve regeneration through the space formed by a chitosan gel sponge. J Biomed Mater Res A 83: 33-40, 2007.

This work is licensed under a Creative Commons Attribution-NonCommercial-NoDerivatives 4.0 International (CC BY-NC-ND 4.0) License. 